\title{
Densidade espectral de potência de sinais transmitidos pelo CSK
}

\author{
Rafael Alves da Costa e Marcio Eisencraft
}

Resumo-Nesse artigo, deriva-se analiticamente expressões para a densidade espectral de potência de sinais transmitidos pelo Chaos Shift Keying, uma modulação digital que utiliza sinais caóticos. Esses resultados são importantes quando se pensa em aplicações práticas desses sinais em sistemas de comunicação e processamento de sinais. Os resultados teóricos são ilustrados por meio de exemplos numéricos.

Palavras-Chave-sinais caóticos, sistemas não lineares, densidade espectral de potência, modulações digitais.

Abstract-In this article, expressions for the power spectral density of signals transmitted by Chaos Shift Keying (CSK) are analytically derived. CSK is a digital modulation that uses chaotic signals. These results are important when considering the practical application of these signals in communication systems and signal processing. Theoretical results are illustrated using numerical examples.

Keywords - chaotic signals, non-linear systems, power spectral density, digital modulations.

\section{INTRODUÇ̃̃o}

Desde o trabalho pioneiro de Pecora e Carroll [1] em que mostrou-se que dois sistemas caóticos poderiam sincronizar, muitos trabalhos foram produzidos sobre sistemas de comunicação baseado em caos (SCBC) [2]-[9].

Especificamente, em implementações digitais de SCBC, a modulação por comutação caótica (CSK - Chaos Shift Keying) [10]-[13] é amplamente conhecida. Ela é uma modulação em que o símbolo a ser transmitido é codificado com os coeficientes de uma combinação linear de sinais caóticos gerados por um ou mais mapas, ou seja, sistemas dinâmicos de tempo discreto [14].

Para viabilizar aplicações práticas da modulação digital que utiliza sinais caóticos é de fundamental importância conhecer e controlar precisamente a densidade espectral de potência (DEP) dos sinais transmitidos.

Nesse artigo, deriva-se expressões analíticas para a DEP de versões do CSK que utilizam mapas lineares por partes. Explora-se aqui o fato de que recentemente foram obtidas expressões analíticas para a DEP de alguns geradores caóticos lineares por partes [15]-[21].

O artigo é organizado da seguinte forma: na Seção II resume-se o funcionamento da modulação CSK que utiliza mapas lineares por partes. Na Seção III deduzem-se a sequência de autocorrelação (SAC) e a DEP dessa modulação. Para alguns casos particulares são mostradas simulações que

Rafael Alves da Costa e Marcio Eisencraft. Departamento de Engenharia de Telecomunicações e Controle da Escola Politécnica da Universidade de São Paulo. E-mail: rcosta@lcs.poli.usp.br e marcioft@usp.br. Este trabalho foi financiado pela Coordenação de Aperfeiçoamento de Pessoal de Nível Superior - Brasil (CAPES) - Código de Financiamento 001. M.E. foi parcialmente financiado por CNPq 311039/2019-7. validam os resultados teóricos na Seção IV. Por fim, na Seção $\mathrm{V}$ tecem-se as conclusões.

\section{MODULAÇÃO CSK}

No CSK binário, para um único mapa, um sinal caótico $x(n)$ é gerado pela equação de diferenças [10, Cap. 6],

$$
x(n)=f(x(n-1))
$$

com $n \in \mathbb{N}$ e $x(0)$ uma condição inicial arbitrária.

Nesse trabalho, toma-se uma partição do intervalo $[-1,1[$ com $r$ intervalos de extremidades $-1<\alpha_{1}<\ldots<\alpha_{r-1}<$ $\alpha_{r}=1$. A cada um desses segmentos associa-se um $\beta_{\ell} \in$ $\{-1,1\}, \ell=1,2, \ldots, r$.

Então, no intervalo $\alpha_{\ell-1} \leq x<\alpha_{\ell}$ o mapa $f(x)$ é definido por

$$
f(x)=\beta_{\ell}\left\{\frac{2 x-\left(\alpha_{\ell}+\alpha_{\ell-1}\right)}{\alpha_{\ell}-\alpha_{\ell-1}}\right\} .
$$

Para facilitar a notação, define-se os vetores coluna $r$ dimensional $\boldsymbol{\alpha}=\left[\begin{array}{llll}\alpha_{1} & \alpha_{2} & \ldots & \alpha_{r}\end{array}\right]^{T}$ e $\boldsymbol{\beta}=\left[\begin{array}{llll}\beta_{1} & \beta_{2} & \ldots & \beta_{r}\end{array}\right]^{T}$ [21].

Na Fig. 1(a) é mostrado um gráfico de um caso particular

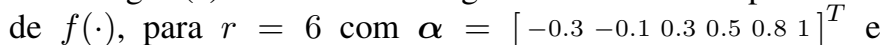
$\boldsymbol{\beta}=\left[\begin{array}{llllll}1 & -1 & 1 & 1 & 1 & -1\end{array}\right]^{T}$. Na Fig. 1(b) mostram-se dois trechos de sinal desse mapa com condições iniciais muito próximas, $x(0)=0.6$ e $x(0)=0.600000001$, deixando-se explícita a dependência sensível às condições iniciais [14].
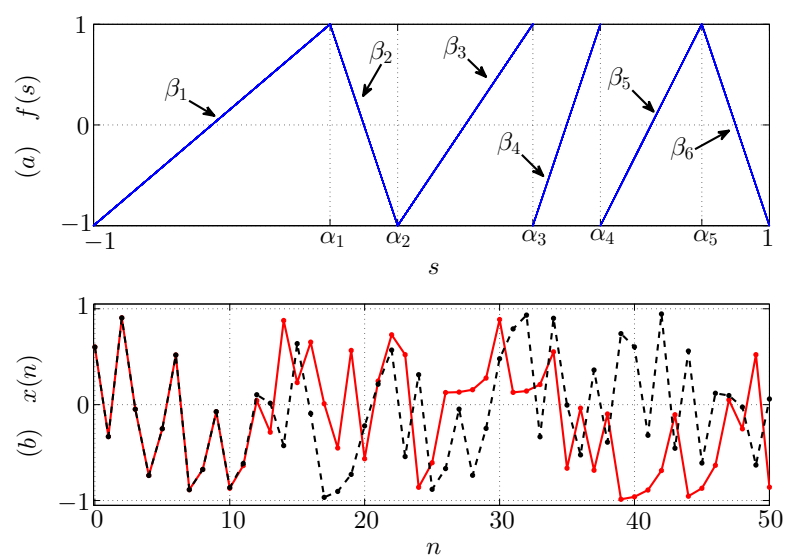

Fig. 1. (a) $\mathrm{O}$ mapa $f(\cdot) \operatorname{com} r=6$ e (b) duas órbitas com $x(0)=0.6$ (linha contínua) e $x(0)=0.600000001$ (linha tracejada).

A mensagem $m(n)$ a ser transmitida é dada por [22]

$$
m(n)=\sum_{i=0}^{\infty} a_{i} \Pi\left(n-i N_{b}\right),
$$


em que, a função porta $\Pi(n)$ é definida por

$$
\Pi(n)=\left\{\begin{array}{l}
1, \quad 0 \leq n<N_{b} \\
0, \quad \text { caso contrário }
\end{array}\right.
$$

e faz-se $a_{i}=c$ para transmitir o bit 1 e $a_{i}=d$ para transmitir o bit 0 .

Para obter o sinal CSK, toma-se o produto de (3) por um sinal caótico $x(n)$ gerado por $f(\cdot)$ de (1) com condição inicial aleatória uniformemente distribuída no domínio do mapa resultando em [10]

$$
s(n)=x(n) m(n) .
$$

Na Fig. 2 tem-se exemplos dos sinais $x(n), m(n)$ e $s(n) . \mathrm{Na}$ Fig. 2(a) mostra-se um sinal caótico gerado pelo mapa (2) com $r=2, \alpha_{1} \triangleq \alpha$ e $\boldsymbol{\beta}=\left[\begin{array}{ll}1 & 1\end{array}\right]^{T}$ e condição inicial aleatória. $\mathrm{Na}$ Fig. 2(b) apresenta-se um trecho da mensagem correspondente a sequência de símbolos $(1,-1,1,1,1,-1,1,-1)$ em que $c=$ 1 e $d=-1$ e na Fig. 2(c) o sinal CSK resultante.
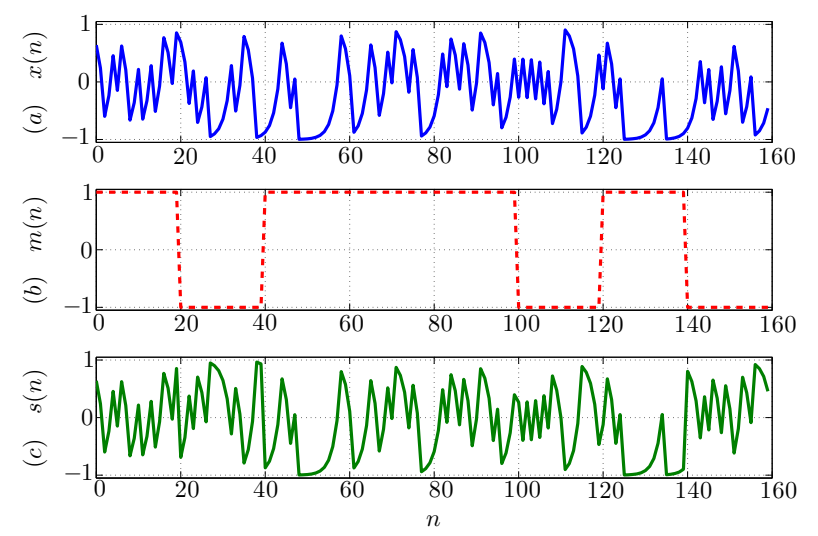

Fig. 2. (a) Sinal caótico gerado pelo mapa (2) com $r=2, \alpha_{1} \triangleq$ $\alpha$ e $\boldsymbol{\beta}=\left[\begin{array}{ll}1 & 1\end{array}\right]^{T}$ com $\alpha=0.05$, (b) mensagem para sequência $(1,-1,1,1,1,-1,1,-1)$ com $N_{b}=20$ e (c) o sinal CSK resultante.

Com relação à demodulação, quando os dois possíveis sinais transmitidos pelo CSK não possuem mesma energia, i.e., $|c| \neq$ $|d|$ é possível utilizar a demodulação não coerente em que utiliza-se o fato de que as energias dos sinais transmitidos são distintas e assim pode-se comparar as energias dos símbolos recebidos no receptor com um limiar de modo a distinguir o bit 0 e o bit 1 [10]. Caso contrário, sugere-se o uso da demodulação coerente em que pode-se utilizar técnicas de sincronização no receptor e recuperar a mensagem transmitida [10].

Um caso particular do CSK é a modulação por comutação liga-desliga caótica (COOK - Chaos On-Off Keying) para a qual $c=1$ e $d=0$ [10]. Um exemplo de modulação COOK é mostrada na Fig. 3.

Na próxima sessão deriva-se expressões analíticas para DEP da modulação CSK.
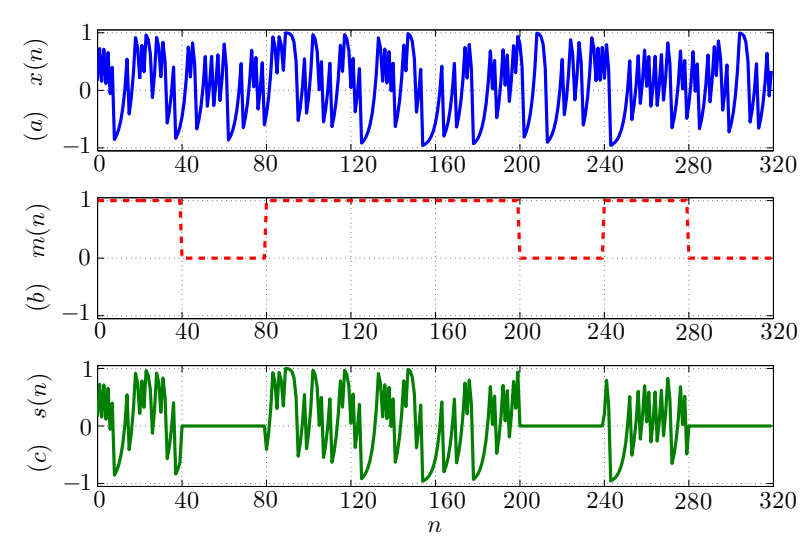

Fig. 3. (a) Sinal caótico gerado pelo mapa (2) $\operatorname{com} r=2, \alpha_{1} \triangleq \alpha$ e $\boldsymbol{\beta}=$ $\left[\begin{array}{ll}1 & 1\end{array}\right]^{T}$ com $\alpha=0.35$, (b) mensagem para sequência $(1,0,1,1,1,0,1,0)$ com $N_{b}=40$ e (c) o sinal COOK resultante.

\section{DENSIDADE ESPECTRAL POTÊNCIA DO CSK}

Para calcular a DEP do CSK, primeiramente, calcula-se a SAC de $s(n)$. Usando-se (5),

$$
\begin{aligned}
R_{s}(k) & =\mathbb{E}[s(n) s(n+k)] \\
& =\mathbb{E}[x(n) x(n+k) m(n) m(n+k)] \\
& =\mathbb{E}[x(n) x(n+k)] \mathbb{E}[m(n) m(n+k)] \\
& =R_{x}(k) R_{m}(k),
\end{aligned}
$$

em que $\mathbb{E}[\cdot]$ é o operador valor esperado e utilizou-se a independência dos processos que caracterizam a mensagem $m(n)$ e o sinal caótico $x(n)$.

A SAC de $x(n)$ é obtida por meio de uma fórmula recursiva para $n$-ésima iteração do mapa linear por partes e resulta em [21]

$$
R_{x}(k)=\frac{1}{3} \psi^{|k|}
$$

com

$$
\psi=\frac{1}{4} \sum_{\ell=1}^{r} \beta_{\ell}\left(\alpha_{\ell}-\alpha_{\ell-1}\right)^{2} .
$$

Sua DEP $\mathcal{X}(\omega)$ é dada pela transformada de Fourier de tempo discreto (TFTD) de (7),

$$
\mathcal{X}(\omega)=\frac{1-\psi^{2}}{3\left(1-2 \cos (\omega) \psi+\psi^{2}\right)} .
$$

$\mathrm{Na}$ Fig. 4 tem-se alguns exemplos de $\mathcal{X}(\omega)$ para o mapa $f(\cdot)$ com parâmetros $\alpha_{1} \triangleq \alpha, \boldsymbol{\beta}=[1-1]^{T}$ e diferentes valores $\alpha$. Esse mapa é conhecido como tenda inclinada [16]. Nota-se que o parâmetro $\alpha$ do mapa controla a forma como a potência é distribuída. A potência pode concentrar-se nas altas, nas baixas frequências ou possuir característica de banda larga.

Pode se mostrar [22], que a SAC da mensagem $m(n)$, descrita em (3), formada por uma sequência binária equiprovável $a_{i}$, resulta em

$$
R_{m}(k)=\lambda^{2}\left(1-\frac{|k|}{N_{b}}\right)+\mu^{2},
$$

com

$$
\lambda=\frac{c-d}{2}
$$




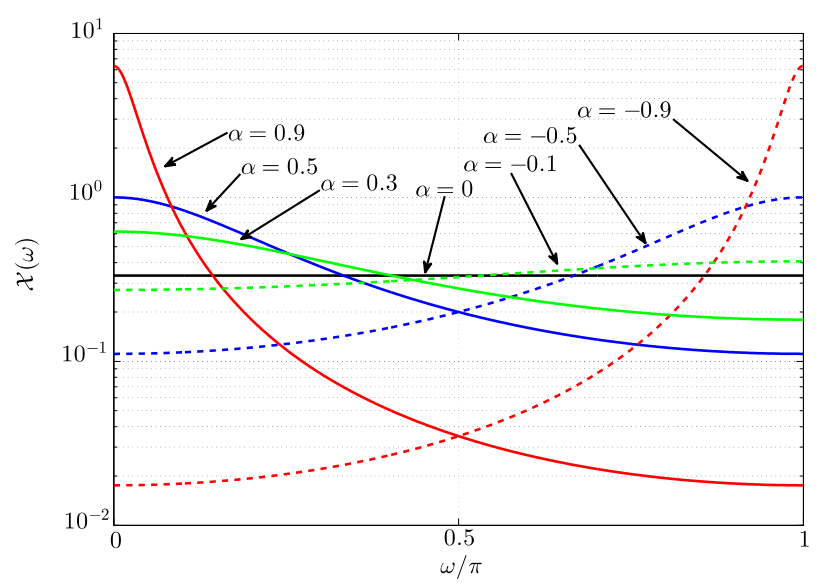

Fig. 4. DEP dos sinais caóticos para diferentes valores de $\alpha$ [16].

e valor médio

$$
\mu=\frac{c+d}{2}
$$

Para obter a DEP de (10) calcula-se a TFTD de $R_{m}(k)$, resultando em

$$
\mathcal{M}(\omega)=\frac{\lambda^{2}}{N_{b}}\left[\frac{\sin \left(\frac{\omega N_{b}}{2}\right)}{\sin \left(\frac{\omega}{2}\right)}\right]^{2}+2 \mu^{2} \delta(\omega) \text { para } \omega \in[-\pi, \pi[
$$

Na Fig. 5 tem-se alguns exemplos de $\mathcal{M}(\omega)$ para diferentes valores $N_{b} \operatorname{com} \lambda=1$ e $\mu=0$.

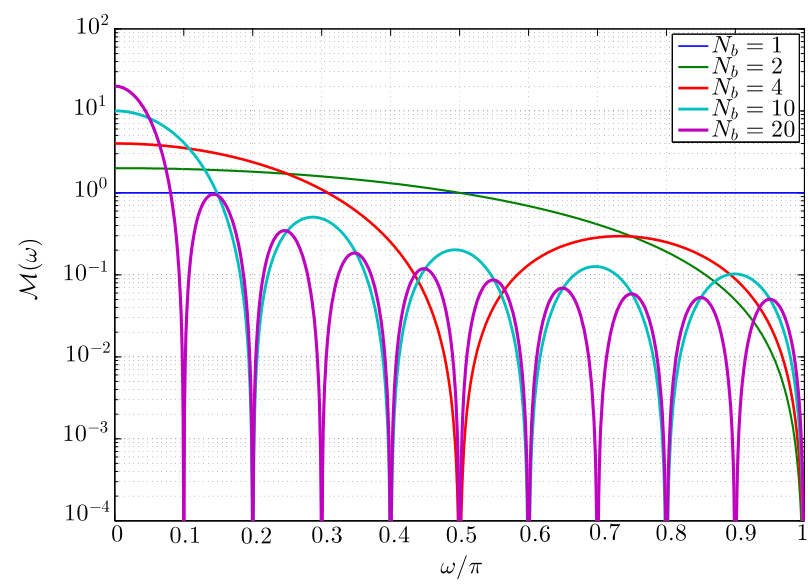

Fig. 5. DEP de $m(n)$ para diferentes valores de $N_{b}$.

Substituindo-se (7) e (10) em (6), obtém-se

$$
R_{s}(k)=\frac{1}{3}\left[\lambda^{2}\left(1-\frac{|k|}{N_{b}}\right)+\mu^{2}\right] \psi^{|k|} .
$$

Finalmente, toma-se a TFTD de (14) para obter a DEP do
CSK

$$
\begin{aligned}
& \mathcal{S}(\omega)=\sum_{k=-\infty}^{\infty} R_{s}(k) e^{-j \omega k} \\
& =\sum_{k=-\left(N_{b}-1\right)}^{N_{b}-1} \frac{1}{3}\left[\lambda^{2}\left(1-\frac{|k|}{N_{b}}\right)+\mu^{2}\right] \psi^{|k|} e^{-j \omega k} \\
& =\frac{\left(\lambda^{2}+\mu^{2}\right)}{3}+\sum_{k=1}^{N_{b}-1} \frac{1}{3}\left[\lambda^{2}\left(1-\frac{k}{N_{b}}\right)+\mu^{2}\right] \psi^{k}\left(e^{-j \omega k}+e^{j \omega k}\right) \\
& =\frac{2}{3} \sum_{k=0}^{N_{b}-1}\left[\lambda^{2}\left(1-\frac{k}{N_{b}}\right)+\mu^{2}\right] \psi^{k} \cos (k \omega)-\frac{\left(\lambda^{2}+\mu^{2}\right)}{3} .
\end{aligned}
$$

Nota-se que esta expressão é geral e possibilita uma diversidade de espectros alcançáveis para modulação CSK utilizandose de geradores de sinais caóticos lineares por partes [15]-[21].

Na próxima seção, exemplifica-se numericamente a expressão geral (15) para alguns casos particulares.

\section{EXEMPLOS NUMÉRICOS}

Nesta seção analisa-se alguns casos particulares da expressão geral da DEP para modulação CSK (15). Investigam-se os casos $N_{b}=1, N_{b}=4, N_{b} \rightarrow \infty$ e o COOK. Nos casos particulares, considera-se o mapa $f(\cdot)$ com parâmetros $r=2$, $\alpha_{1} \triangleq \alpha$ e $\boldsymbol{\beta}=[1-1]^{T}$, ou seja, o mapa tenda inclinada [16].

De (8), obtêm-se

$$
\begin{aligned}
\psi & =\frac{1}{4} \sum_{\ell=1}^{2} \beta_{\ell}\left(\alpha_{\ell}-\alpha_{\ell-1}\right)^{2} \\
& =\frac{1}{4}\left(\beta_{1}\left(\alpha_{1}-\alpha_{0}\right)^{2}+\beta_{2}\left(\alpha_{2}-\alpha_{1}\right)^{2}\right) \\
& =\frac{1}{4}\left((\alpha+1)^{2}-(1-\alpha)^{2}\right)=\alpha .
\end{aligned}
$$

Substituindo-se (16) em (15) tem-se

$$
\mathcal{S}(\omega)=\frac{2}{3} \sum_{k=0}^{N_{b}-1}\left(1-\frac{k}{N_{b}}\right) \alpha^{k} \cos (k \omega)-\frac{1}{3} .
$$

Na Fig. 6 ilustra-se (17) com $\alpha=-0.5$ para alguns valores de $N_{b}$. Nota-se que para esse valor de parâmetro, a DEP da modulação CSK aproxima-se da DEP de (9) conforme $N_{b}$ cresce. Quando $N_{b}=1$, o espectro é plano. Claramente, a DEP concentra-se nas altas frequências para valores de $N_{b} \neq$ 1. Já na Fig. 7, com $\alpha=0.5$, a DEP concentra-se nas baixas frequências apresentando simetria em relação à Fig. 6 .

\section{A. Caso: CSK com $N_{b}=1$}

Nesse caso a somatória (15) apresenta apenas uma parcela e

$$
\mathcal{S}(\omega)=\left(\lambda^{2}+\mu^{2}\right) / 3
$$

Nota-se que independente dos parâmetros escolhidos para o mapa $f(\cdot)$ a DEP da modulação CSK resulta em um espectro plano. De (18), obtém-se $\mathcal{S}(\omega)=1 / 3$, ou seja, o sinal transmitido comporta-se em termos espectrais como um ruído branco. 


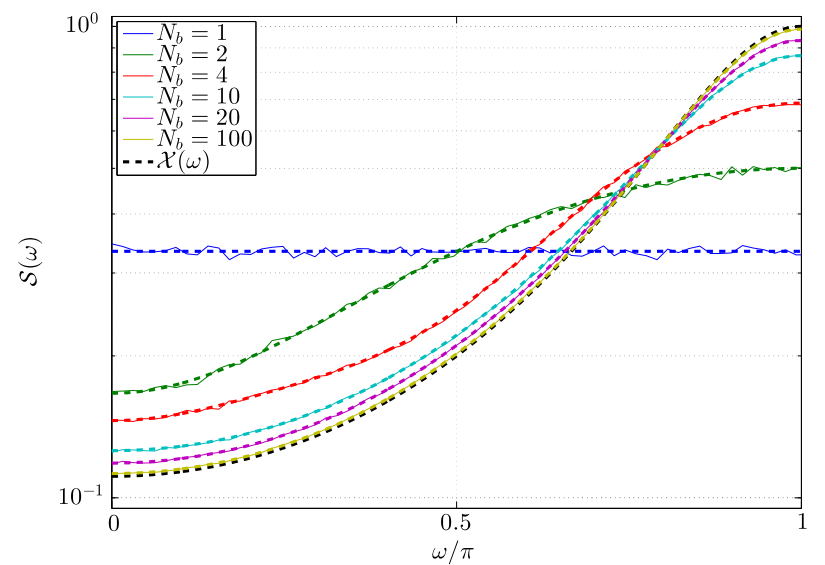

Fig. 6. $\mathcal{S}(\omega)$ para diferentes valores de $N_{b}$ com $\alpha=-0.5$. Em linha contínua estão as curvas obtidas via simulação numérica e curvas analíticas em linha tracejada.

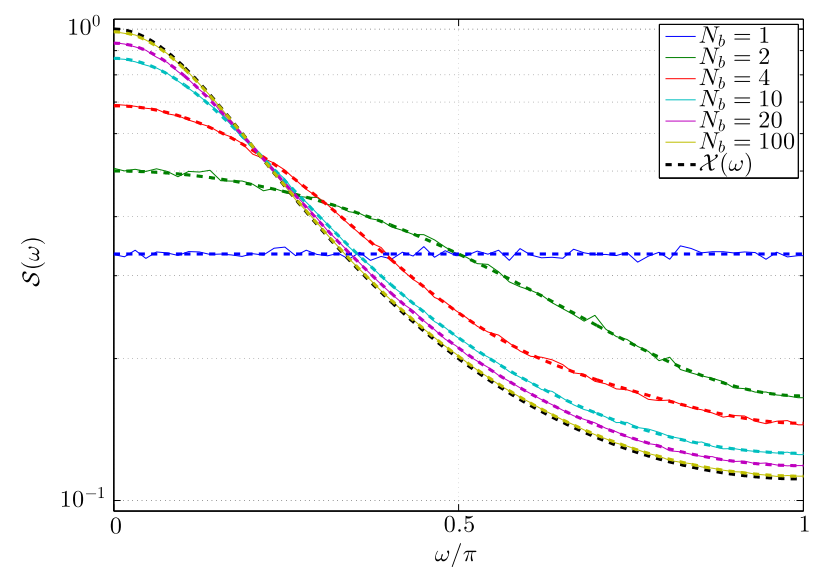

Fig. 7. $\mathcal{S}(\omega)$ para diferentes valores de $N_{b}$ com $\alpha=0.5$. Em linha contínua estão as curvas obtidas via simulação numérica e curvas analíticas em linha tracejada.

\section{B. Caso: CSK com $N_{b}=4$}

De (17), para $N_{b}=4$ tem-se

$$
\begin{aligned}
\mathcal{S}(\omega) & =\frac{2}{3} \sum_{k=0}^{3}\left(1-\frac{k}{4}\right) \alpha^{k} \cos (k \omega)-\frac{1}{3} \\
& =\frac{1}{3}+\left(\frac{\alpha}{2}\right) \cos (\omega)+\left(\frac{\alpha^{2}}{3}\right) \cos (2 \omega) \\
& +\left(\frac{\alpha^{3}}{6}\right) \cos (3 \omega) .
\end{aligned}
$$

Nas Fig. 8 é apresentado a DEP de (19) para alguns valores de $\alpha$. Quando o parâmetro $\alpha$ é positivo, a potência concentra-se nas baixas frequências. Quando $\alpha$ é negativo a DEP concentra-se nas altas frequências. Nota-se que esse resultado é parecido com o exemplo da Fig. 4, entretanto, $\mathcal{S}(\omega)$ possui uma largura de banda maior e uma tendência de sua DEP aproximar-se de $\mathcal{M}(\omega)$ quando o parâmetro $|\alpha| \rightarrow 1$.

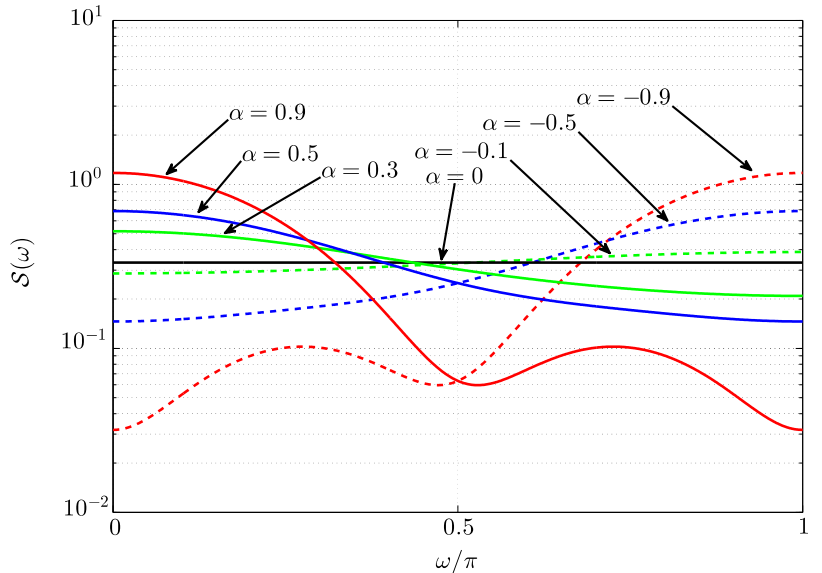

Fig. 8. DEP do CSK com $N_{b}=4$ para diferentes valores de $\alpha$.

C. Caso: CSK com $N_{b} \rightarrow \infty$

Neste caso, utilizando-se (15)

$$
\begin{aligned}
\mathcal{S}(\omega) & =\frac{2}{3} \sum_{k=0}^{\infty} \psi^{k} \cos (k \omega)-\frac{1}{3} \\
& =\sum_{k=0}^{\infty} \frac{1}{3} \psi^{k}\left(e^{-j \omega k}+e^{j \omega k}\right)-\frac{1}{3} \\
& =\frac{1}{3}\left[\left(\sum_{k=0}^{\infty} \psi^{k} e^{j \omega k}\right)+\left(\sum_{k=0}^{\infty} \psi^{k} e^{-j \omega k}\right)-1\right] \\
& =\frac{1}{3}\left[\frac{1}{1-\psi e^{j \omega}}+\frac{1}{1-\psi e^{-j \omega}}-1\right] \\
& =\frac{1}{3} \frac{1-\psi^{2}}{1-\psi\left(e^{j \omega}+e^{-j \omega}\right)+\psi^{2}} \\
& =\frac{1-\psi^{2}}{3\left(1-2 \psi \cos (\omega)+\psi^{2}\right)}
\end{aligned}
$$

Comparando-se com (9), nota-se que obtém-se novamente a DEP do sinal caótico puro, como esperado. Além disso, utilizando-se (16) obtém-se a DEP do mapa tenda inclinada [16].

\section{D. $\mathrm{COOK}$}

Outro caso particular de (15) é quando o sinal digital possui $c=1$ e $d=0$. Nesse caso, utilizando-se (10)-(12) a SAC do sinal digital da modulação COOK resulta em

$$
R_{m}(k)=\frac{1}{4}\left(1-\frac{|k|}{N_{b}}\right)+\frac{1}{4}
$$

Consequentemente, usando-se (21) em (15) tem-se

$$
\mathcal{S}(\omega)=\frac{2}{3} \sum_{k=0}^{N_{b}-1}\left[\frac{1}{4}\left(1-\frac{k}{N_{b}}\right)+\frac{1}{4}\right] \psi^{k} \cos (k \omega)-\frac{1}{6} \text {. }
$$

Nota-se que de (18), a primeira parcela em (22) é dada por $\mathcal{S}(\omega)=1 / 6$.

Substituindo-se (16) em (22), obtêm-se

$$
\mathcal{S}(\omega)=\frac{2}{3} \sum_{k=0}^{N_{b}-1} \frac{1}{4}\left[\left(1-\frac{k}{N_{b}}\right)+1\right] \alpha^{k} \cos (k \omega)-\frac{1}{6} .
$$


Na Fig. 9 ilustra-se (23) com $\alpha=-0.5$. Nota-se que a DEP dos sinais modulados COOK são idênticas às da modulação CSK concentrando-se nas altas frequências, havendo apenas atenuação de $1 / 2$. Já na Fig. 10 , com $\alpha=0.5$, a DEP concentra-se nas baixas frequências novamente apresentando simetria em relação à Fig. 9 .

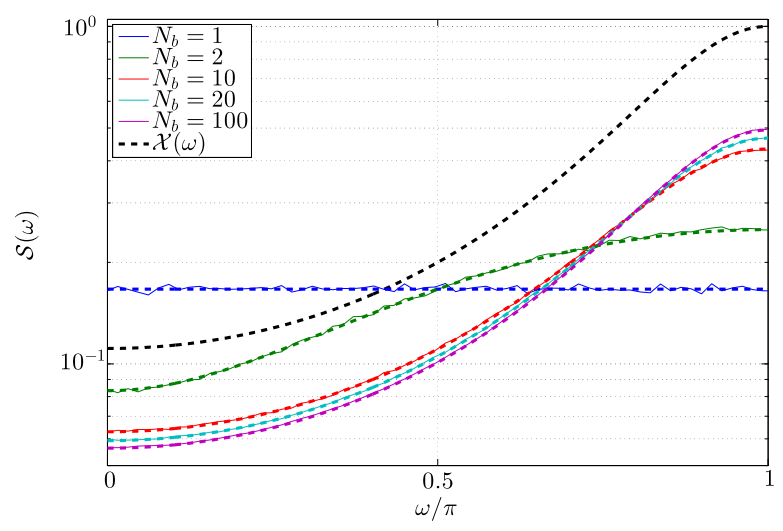

Fig. 9. $\mathcal{S}(\omega)$ para diferentes valores de $N_{b}$ com $\alpha=-0.5$. Em linha contínua estão as curvas obtidas via simulação numérica e curvas analíticas em linha tracejada.

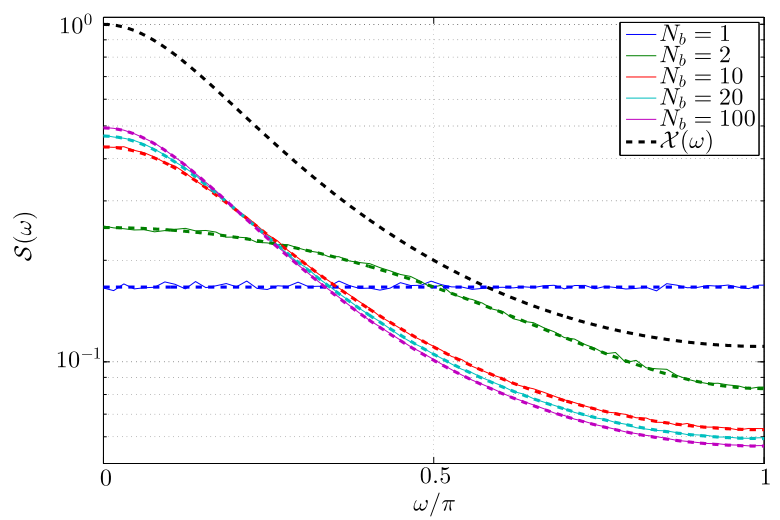

Fig. 10. $\mathcal{S}(\omega)$ para diferentes valores de $N_{b}$ com $\alpha=0.5$. Em linha contínua estão as curvas obtidas via simulação numérica e curvas analíticas em linha tracejada.

\section{Conclusões}

Quando se pensa na aplicação prática da modulação CSK, na área de Telecomunicações, é de fundamental importância conhecer e controlar as características espectrais dos sinais gerados por ela. Nesse artigo, deduziu-se a SAC e a DEP da modulação CSK que utiliza-se de geradores caóticos lineares por partes. Em particular, foram analisados, teórica e numericamente, alguns casos particulares dessa modulação. Esses resultados podem ser úteis em projetos de SCBC.

Como continuação desse trabalho pretende-se estender o estudo para outras modulações digitais que utilizam sinais caóticos, como por exemplo, a comutação caótica diferencial (DCSK - Differential Chaos Shift Keying) [10]. Além disso, pretende-se utilizar Universal Software Radio Peripherical (USRP) para desenvolver testes experimentais de SCBC [23].

\section{REFERÊNCIAS}

[1] L. M. Pecora and T. L. Carroll, "Synchronization in chaotic systems," Physical Review Letters, vol. 64, no. 8, pp. 821-824, feb 1990.

[2] S. Hayes, C. Grebogi, and E. Ott, "Communicating with chaos," Physical Review Letters, vol. 70, no. 20, pp. 3031-3034, may 1993.

[3] G. Kolumban, M. Kennedy, and L. Chua, "The role of synchronization in digital communications using chaos. $\mathrm{i}$. fundamentals of digital communications," vol. 44, no. 10, pp. 927-936, Oct 1997.

[4] H. Dedieu, M. Kennedy, and M. Hasler, "Chaos shift keying: modulation and demodulation of a chaotic carrier using self-synchronizing chua's circuits," Circuits and Systems II: Analog and Digital Signal Processing, IEEE Transactions on, vol. 40, no. 10, pp. $634-642$, oct 1993. [Online]. Available: http://dx.doi.org/10.1109/82.246164

[5] M. Eisencraft and G. Abib, "Sobre o desempenho em canal com ruído de um sistema de comunicação baseado em caos," in Anais de XXXI Simpósio Brasileiro de Telecomunicações, Sociedade Brasileira de Telecomunicações. Sociedade Brasileira de Telecomunicações, 2013.

[6] H.-P. Ren, M. S. Baptista, and C. Grebogi, "Wireless communication with chaos," Physical Review Letters, vol. 110, no. 18, p. 184101, apr 2013.

[7] G. Kaddoum, "Wireless chaos-based communication systems: A comprehensive survey," IEEE Access, vol. 4, pp. 2621-2648, 2016.

[8] H.-P. Ren, C. Bai, J. Liu, M. S. Baptista, and C. Grebogi, "Experimental validation of wireless communication with chaos," Chaos: An Interdisciplinary Journal of Nonlinear Science, vol. 26, no. 8, p. 083117, aug 2016.

[9] C. E. C. Souza, D. P. B. Chaves, and C. Pimentel, "Digital communication systems based on three-dimensional chaotic attractors," IEEE Access, vol. 7, pp. 10 523-10 532, 2019.

[10] M. P. Kennedy, G. Setti, and R. Rovatti, Eds., Chaotic Electronics In Telecommunications. Boca Raton, FL, USA: CRC Press, 2000.

[11] F. C. M. Lau and C. K. Tse, Chaos-Based Digital Communication Systems. Berlin: Springer-Verlag GmbH, 2003.

[12] P. Stavroulakis, Ed., Chaos Applications in Telecommunications. Boca Raton, FL, USA: CRC Press, 2005.

[13] M. Eisencraft, R. R. F. Attux, and R. Suyama, Eds., Chaotic Signals in Digital Communications (Electrical Engineering \& Applied Signal Processing Series). CRC Press, 2013.

[14] K. T. Alligood, T. D. Sauer, and J. A. Yorke, Chaos, ser. Textbooks in Mathematical Sciences. Springer New York, 2000.

[15] D. M. Kato and M. Eisencraft, "Caracterização espectral de sinais caóticos: resultados analíticos," in Anais do XXVI Simpósio Brasileiro de Telecomunicações. Rio de Janeiro: Sociedade Brasileira de Telecomunicações, 2008, pp. 1-6.

[16] M. Eisencraft, D. Kato, and L. Monteiro, "Spectral properties of chaotic signals generated by the skew tent map," Signal Processing, vol. 90, no. 1, pp. 385-390, jan 2010.

[17] K. Feltekh, D. Fournier-Prunaret, and S. Belghith, "Analytical expressions for power spectral density issued from one-dimensional continuous piecewise linear maps with three slopes," Signal Processing, vol. 94, no. 0, pp. 149-157, jan 2014.

[18] R. A. da Costa, M. B. Loiola, and M. Eisencraft, "Caracterização espectral de sinais caóticos gerados pelo mapa de bernoulli com 2 e 3 segmentos," in Anais de XXXIII Simpósio Brasileiro de Telecomunicações. Sociedade Brasileira de Telecomunicações, 2015, pp. 1-5.

[19] R. A. Costa, M. B. Loiola, and M. Eisencraft, "Correlation and spectral properties of chaotic signals generated by a piecewise-linear map with multiple segments," Signal Processing, vol. 133, pp. 187-191, apr 2017.

[20] A. Sahnoune and D. Berkani, "On the correlation of chaotic signals generated by multimodal skew tent map," Signal, Image and Video Processing, vol. 12, no. 7, pp. 1273-1278, mar 2018.

[21] R. A. da Costa and M. Eisencraft, "Spectral characteristics of a general piecewise linear chaotic signal generator," Communications in Nonlinear Science and Numerical Simulation, vol. 72, pp. 441-448, jun 2019.

[22] S. S. Haykin, Communication systems, 4th ed. New York: Wiley, 2001.

[23] G. Kolumban, T. I. Krebesz, and F. C. M. Lau, "Theory and application of software defined electronics: Design concepts for the next generation of telecommunications and measurement systems," IEEE Circuits and Systems Magazine, vol. 12, no. 2, pp. 8-34, Secondquarter 2012. 